\title{
Two sufficient conditions for fractional $k$-deleted graphs
}

\author{
Xiangyang Lv
}

\begin{abstract}
Let $G$ be a graph, and $k$ a positive integer. A fractional $k$-factor is a way of assigning weights to the edges of a graph $G$ (with all weights between 0 and 1 ) such that for each vertex the sum of the weights of the edges incident with that vertex is $k$. A graph $G$ is a fractional $k$-deleted graph if $G-e$ has a fractional $k$-factor for each $e \in E(G)$. In this paper, we obtain some sufficient conditions for graphs to be fractional $k$-deleted graphs in terms of their minimum degree and independence number. Furthermore, we show the results are best possible in some sense.
\end{abstract}

\section{Introduction}

The graphs considered here will be finite undirected graphs without loops or multiple edges. Let $G$ be a graph with vertex set $V(G)$ and edge set $E(G)$. For any $x \in V(G)$, we denote by $d_{G}(x)$ the degree of $x$ in $G$ and by $N_{G}(x)$ the set of vertices adjacent to $x$ in $G$. For $S \subseteq V(G)$, we denote by $G[S]$ the subgraph of $G$ induced by $S$ and by $G-S$ the subgraph obtained from $G$ by deleting vertices in $S$ together with the edges incident to vertices in $S$. Let $S$ and $T$ be two disjoint subsets of $V(G)$, we denote by $e_{G}(S, T)$ the number of edges with one end in $S$ and the other end in $T$. A subset $S$ of $V(G)$ is called an independent set of $G$ if every edge of $G$ is incident with at most one

Key Words: operations research, independence number, minimum degree, fractional $k$-factor, fractional $k$-deleted graph.

2010 Mathematics Subject Classification: 05C70, 90B99.

Received: January, 2011.

Accepted: October, 2011. 
vertex of $S$. We use $\alpha(G)$ and $\delta(G)$ to denote the independence number and minimum degree of $G$, respectively.

Let $k$ be a positive integer. Then a spanning subgraph $F$ of $G$ is called a $k$-factor if $d_{F}(x)=k$ for each $x \in V(G)$. If $k=1$, then a $k$-factor is simply called a 1 -factor. A fractional $k$-factor is a way of assigning weights to the edges of a graph $G$ (with all weights between 0 and 1) such that for each vertex the sum of the weights of the edges incident with that vertex is $k$. If $k=1$, then a fractional $k$-factor is a fractional 1-factor. A graph $G$ is a fractional $k$-deleted graph if $G-e$ has a fractional $k$-factor for each $e \in E(G)$. If $k=1$, then a fractional $k$-deleted graph is a fractional 1-deleted graph. If $G_{1}$ and $G_{2}$ are disjoint graphs, then the union is denoted by $G_{1} \cup G_{2}$ and the join by $G_{1} \bigvee G_{2}$. The other terminologies and notations not given here can be found in [1].

Many authors have investigated graph factors $[6,7,11,12]$. Many authors have investigated fractional $k$-factors $[2,5,8,13]$ and fractional $k$-deleted graphs $[3,9,10]$. The following results on $k$-factors, fractional $k$-factors and fractional $k$-deleted graphs are known.

Theorem 1. ${ }^{[6]}$ Let $k \geq 2$ be an integer and $G$ a graph with $n$ vertices. Assume that if $k$ is odd, then $n$ is even and $G$ is connected. Let $G$ satisfy

$$
\begin{gathered}
n>4 k+1-4 \sqrt{k+2}, \\
\delta(G) \geq \frac{(k-1)(n+2)}{2 k-1} \quad \text { and } \\
\delta(G)>\frac{1}{2 k-2}((k-2) n+2 \alpha(G)-2) .
\end{gathered}
$$

Then $G$ has a $k$-factor.

Theorem 2. ${ }^{[13]}$ Let $k \geq 2$ be an even integer and $G$ a graph of order $n$ with $n>4 k+1-4 \sqrt{k+2}$. If

$$
\begin{gathered}
\delta(G) \geq \frac{(k-1)(n+2)}{2 k-1} \quad \text { and } \\
\delta(G)>\frac{1}{2 k-2}((k-2) n+2 \alpha(G)-2),
\end{gathered}
$$

then $G$ has a fractional $k$-factor.

Theorem 3. ${ }^{[13]}$ Let $k \geq 3$ be an odd integer and $G$ a graph of order $n$ with $n \geq 4 k-5$. If

$$
\delta(G)>\frac{(k-1)(n+2)}{2 k-1} \quad \text { and }
$$




$$
\delta(G)>\frac{1}{2 k-2}((k-2) n+2 \alpha(G)-1),
$$

then $G$ has a fractional $k$-factor.

Theorem 4. ${ }^{[10]}$ Let $k \geq 2$ be an integer, and let $G$ be a graph of order $n$ with $n \geq 4 k-5$. If

$$
\operatorname{bind}(G)>\frac{(2 k-1)(n-1)}{k(n-2)},
$$

then $G$ is a fractional $k$-deleted graph.

In this paper, we shall proceed to research the fractional $k$-deleted graphs and give some new sufficient conditions for graphs to be fractional $k$-deleted graphs in terms of their minimum degree and independence number. Our main results are the following theorems which are some extensions of Theorem 1, Theorem 2 and Theorem 3.

Theorem 5. Let $k \geq 2$ be an even integer and $G$ a graph of order $n$ with $n>4 k+1-4 \sqrt{k}$. If

$$
\begin{gathered}
\delta(G)>\frac{(k-1)(n+2)+1}{2 k-1} \quad \text { and } \\
\delta(G)>\frac{(k-2) n+2 \alpha(G)}{2 k-2},
\end{gathered}
$$

then $G$ is a fractional $k$-deleted graph.

Theorem 6. Let $k \geq 3$ be an odd integer and $G$ a graph of order $n$ with $n>4 k+1-4 \sqrt{k-1}$. If

$$
\begin{gathered}
\delta(G)>\frac{(k-1)(n+2)+2}{2 k-1} \text { and } \\
\delta(G)>\frac{(k-2) n+2 \alpha(G)+1}{2 k-2},
\end{gathered}
$$

then $G$ is a fractional $k$-deleted graph.

\section{The Proofs of Main Theorems}

In order to prove our main theorems, we depend heavily on the following results. 
Lemma 2.1. ${ }^{[4]}$ A graph $G$ is a fractional $k$-deleted graph if and only if for any $S \subseteq V(G)$ and $T=\left\{x: x \in V(G) \backslash S, d_{G-S}(x) \leq k\right\}$

$$
\delta_{G}(S, T)=k|S|+d_{G-S}(T)-k|T| \geq \varepsilon(S, T),
$$

where $d_{G-S}(T)=\sum_{x \in T} d_{G-S}(x)$ and $\varepsilon(S, T)$ is defined as follows,

$$
\varepsilon(S, T)=\left\{\begin{array}{l}
2, \quad \text { if } T \text { is not independent, } \\
1, \quad \text { if } T \text { is independent, and } e_{G}(T, V(G) \backslash(S \cup T)) \geq 1, \\
0, \quad \text { otherwise. }
\end{array}\right.
$$

Lemma 2.2. ${ }^{[3]}$ Let $a, b$ and $c$ be integers such that $a \geq 2,2 \leq b \leq a-1$, $c=0$ or 1 , and let $x$ and $y$ be nonnegative integers. Suppose that

$$
x \leq \frac{(a-b) y+c}{2 a-b}
$$

and

$$
x>\frac{(a-1)(y+2)+1+c}{2 a-1}-h .
$$

Then $y \leq 4 a+1-4 \sqrt{a-c}$.

In the following, we shall prove our main theorems.

Proof of Theorem 5. Let $G$ be a graph satisfying the hypothesis of Theorem 5, we prove the theorem by contradiction. Suppose that $G$ is not a fractional $k$-deleted graph. Then by Lemma 2.1, there exists a subset $S$ of $V(G)$ such that

$$
\delta_{G}(S, T)=k|S|+d_{G-S}(T)-k|T| \leq \varepsilon(S, T)-1,
$$

where $T=\left\{x: x \in V(G) \backslash S, d_{G-S}(x) \leq k\right\}$.

If $T=\emptyset$, then $\varepsilon(S, T)=0$. Combining this with (1), we have $-1 \geq$ $\delta_{G}(S, T)=k|S| \geq 0$, a contradiction. Therefore, $T \neq \emptyset$. In the following, we define

$$
h=\min \left\{d_{G-S}(x): x \in T\right\}
$$

and choose a vertex $x_{1} \in T$ such that

$$
d_{G-S}\left(x_{1}\right)=h .
$$

Obviously, $0 \leq h \leq k$ and $\delta(G) \leq d_{G}\left(x_{1}\right) \leq d_{G-S}\left(x_{1}\right)+|S|=h+|S|$. Thus, we obtain

$$
|S| \geq \delta(G)-h .
$$

We shall consider three cases by the value of $h$ and derive contradictions. 
Case 1. $h=0$.

Set $X=\left\{x \in T: d_{G-S}(x)=0\right\}, Y=\left\{x \in T: d_{G-S}(x)=1\right\}, Y_{1}=\{x \in$ $\left.Y: N_{G-S}(x) \subseteq T\right\}$ and $Y_{2}=Y-Y_{1}$. Then the graph induced by $Y_{1}$ in $G-S$ has maximum degree at most 1 . Let $Z$ be a maximum independent set of the graph. Obviously, $|Z| \geq \frac{1}{2}\left|Y_{1}\right|$. According to the definitions, $X \cup Z \cup Y_{2}$ is an independent set of $G$. Therefore, we have

$$
\alpha(G) \geq|X|+|Z|+\left|Y_{2}\right| \geq|X|+\frac{1}{2}\left|Y_{1}\right|+\frac{1}{2}\left|Y_{2}\right|=|X|+\frac{1}{2}|Y| .
$$

Using (1), (3) and $|S|+|T| \leq n$, we obtain

$$
\begin{aligned}
1 & \geq \varepsilon(S, T)-1 \geq \delta_{G}(S, T)=k|S|+d_{G-S}(T)-k|T| \\
& =k|S|+d_{G-S}(T \backslash(X \cup Y))-k|T|+|Y| \\
& \geq k|S|+2|T-(X \cup Y)|-k|T|+|Y| \\
& =k|S|+2|T|-k|T|-2|X|-|Y| \\
& =k|S|-(k-2)|T|-2\left(|X|+\frac{1}{2}|Y|\right) \\
& \geq k|S|-(k-2)(n-|S|)-2\left(|X|+\frac{1}{2}|Y|\right) \\
& =(2 k-2)|S|-(k-2) n-2\left(|X|+\frac{1}{2}|Y|\right) \\
& \geq(2 k-2)|S|-(k-2) n-2 \alpha(G),
\end{aligned}
$$

that is,

$$
(2 k-2)|S|-(k-2) n-2 \alpha(G) \leq 1 .
$$

Note that $k$ is even. Therefore, the left-hand side of (4) is even. Thus, we obtain

$$
(2 k-2)|S|-(k-2) n-2 \alpha(G) \leq 0,
$$

which implies

$$
|S| \leq \frac{(k-2) n+2 \alpha(G)}{2 k-2} .
$$

On the other hand, from $(2), h=0$ and $\delta(G)>\frac{(k-2) n+2 \alpha(G)}{2 k-2}$, we get

$$
|S| \geq \delta(G)-h>\frac{(k-2) n+2 \alpha(G)}{2 k-2},
$$

which contradicts (5).

Case 2. $1 \leq h \leq k-1$.

Claim 1. ${ }^{[12]}|S| \leq \frac{(k-h) n}{2 k-h}$. 
On the other hand, by $(2)$ and $\delta(G)>\frac{(k-1)(n+2)+1}{2 k-1}$, we get

$$
|S| \geq \delta(G)-h>\frac{(k-1)(n+2)+1}{2 k-1}-h .
$$

If $h=1$, then (6) contradicts Claim 1. In the following, we assume that $2 \leq h \leq k-1$. Applying Lemma 2.2 with $a=k, b=h, c=0, x=|S|$ and $y=n$, we get

$$
n \leq 4 k+1-4 \sqrt{k},
$$

which contradicts the hypothesis that $n>4 k+1-4 \sqrt{k}$.

Case 3. $h=k$.

It is easy to see that $4 k+1-4 \sqrt{k} \geq 2 k-1$. Hence, we have $n>2 k-1$. Thus, we obtain

$$
\delta(G)>\frac{(k-1)(n+2)+1}{2 k-1}=\frac{(k-1) n}{2 k-1}+1>k .
$$

In terms of the integrity of $\delta(G)$, we obtain

$$
\delta(G) \geq k+1
$$

Claim 2. $S \neq \emptyset$.

Proof. If $S=\emptyset$, then by (1) and (7) we have

$$
\begin{aligned}
\varepsilon(S, T)-1 & \geq \delta_{G}(S, T)=k|S|+d_{G-S}(T)-k|T| \\
& =d_{G}(T)-k|T| \geq \delta(G)|T|-k|T| \geq|T| \geq \varepsilon(S, T)
\end{aligned}
$$

it is a contradiction. The proof of Claim 2 is complete.

According to Claim $2, h=k$ and $k \geq 2$, we obtain

$$
\begin{aligned}
\delta_{G}(S, T) & =k|S|+d_{G-S}(T)-k|T| \\
& \geq k|S|+h|T|-k|T|=k|S| \geq k \geq 2 \geq \varepsilon(S, T),
\end{aligned}
$$

which contradicts (1).

From the contradictions above, we deduce that $G$ is a fractional $k$-deleted graph. This completes the proof of Theorem 5 .

The proof of Theorem 6 is quite similar to that of Theorem 5 and is omitted.

\section{Remarks}

Remark 1. We now show that the conditions $\delta(G)>\frac{(k-1)(n+2)+1}{2 k-1}$ and $\delta(G)>\frac{(k-2) n+2 \alpha(G)}{2 k-2}$ in Theorem 5 are best possible. Let $k \geq 2$ be an integer 
and $G=K_{2 k-2} \bigvee k K_{2}$. We denote by $n$ the order of the graph $G$. Then $n=4 k-2>4 k+1-4 \sqrt{k}$ and $\alpha(G)=k$. Thus, we have $\delta(G)=2 k-1=$ $\frac{(k-1)(n+2)+1}{2 k-1}$ and $\delta(G)=2 k-1=\frac{(2 k-1)(2 k-2)}{2 k-2}=\frac{4 k^{2}-6 k+2}{2 k-2}>\frac{4 k^{2}-8 k+4}{2 k-2}=$ $\frac{4 k^{2}-10 k+4+2 k}{2 k-2}=\frac{(k-2)(4 k-2)+2 k}{2 k-2}=\frac{(k-2) n+2 \alpha(G)}{2 k-2}$. Let $S=V\left(K_{2 k-2}\right), T=$ $V\left(k \stackrel{2 k-2}{K_{2}}\right.$. Then $|S|=2 k-2,|T|=2 k$, and $d_{G-S}(T)=2 k$. Since $T=V\left(k K_{2}\right)$ is not independent, $\varepsilon(S, T)=2$. Thus, we get

$$
\begin{aligned}
\delta_{G}(S, T) & =k|S|+d_{G-S}(T)-k|T| \\
& =k(2 k-2)+2 k-k \cdot 2 k \\
& =0<2=\varepsilon(S, T) .
\end{aligned}
$$

Then by Lemma 2.1, $G$ is not a fractional $k$-deleted graph. In the above sense, the condition $\delta(G)>\frac{(k-1)(n+2)+1}{2 k-1}$ in Theorem 5 is best possible.

Let $k \geq 2$ is even. Obviously, $\frac{k}{2}$ is a positive integer. Put $G=K_{3 k-1} \bigvee$ $\left(2 k K_{1} \bigcup \frac{k}{2} K_{2}\right)$. We use $n$ to denote the order of the graph $G$. Then $n=$ $6 k-1>4 k+1-4 \sqrt{k}$ and $\alpha(G)=2 k+\frac{k}{2}=\frac{5 k}{2}$. Thus, $\delta(G)=3 k-$ $1=\frac{(3 k-1)(2 k-2)}{2 k-2}=\frac{6 k^{2}-8 k+2}{2 k-2}=\frac{(k-2)(6 k-1)+5 k}{2 k-2}=\frac{(k-2) n+2 \alpha(G)}{2 k-2}$ and $\delta(G)=$ $3 k-1=\frac{(3 k-1)(2 k-1)}{2 k-1}=\frac{(k-1)(6 k+1)+2}{2 k-1}=\frac{(k-1)(n+2)+2}{2 k-1}>\frac{(k-1)(n+2)+1}{2 k-1}$. Let $S=V\left(K_{3 k-1}\right), T=V\left(2 k K_{1} \bigcup \frac{k}{2} K_{2}\right)$. Clearly, $|S|=3 k-1,|T|=3 k$, and $d_{G-S}(T)=k$. Since $T=V\left(2 k K_{1} \bigcup \frac{k}{2} K_{2}\right)$ is not independent, $\varepsilon(S, T)=2$. Thus, we have

$$
\begin{aligned}
\delta_{G}(S, T) & =k|S|+d_{G-S}(T)-k|T| \\
& =k(3 k-1)+k-k \cdot 3 k \\
& =0<2=\varepsilon(S, T) .
\end{aligned}
$$

Then by Lemma 2.1, $G$ is not a fractional $k$-deleted graph. In the above sense, the condition $\delta(G)>\frac{(k-2) n+2 \alpha(G)}{2 k-2}$ in Theorem 5 is best possible.

Remark 2. We show that the conditions $\delta(G)>\frac{(k-1)(n+2)+2}{2 k-1}$ and $\delta(G)>$ $\frac{(k-2) n+2 \alpha(G)+1}{2 k-2}$ in Theorem 6 are best possible. Let $k \geq 3$ be an odd integer and $G=K_{3 k-2} \bigvee \frac{3 k+1}{2} K_{2}$. Clearly, $\frac{3 k+1}{2}$ is a positive integer. We denote by $n$ the order of the graph $G$. Then $n=6 k-1>4 k+1-4 \sqrt{k-1}$ and $\alpha(G)=\frac{3 k+1}{2}$. Thus, we have $\delta(G)=3 k-1=\frac{(3 k-1)(2 k-1)}{2 k-1}=\frac{6 k^{2}-5 k+1}{2 k-1}=\frac{(k-1)(6 k+1)+2}{2 k-1}=$ $\frac{(k-1)(n+2)+2}{2 k-1}$ and $\delta(G)=3 k-1=\frac{(3 k-1)(2 k-2)}{2 k-2}=\frac{6 k^{2}-8 k+2}{2 k-2}>\frac{6 k^{2}-10 k+4}{2 k-2}=$ $\frac{(k-2)(6 k-1)+3 k+2}{2 k-2}=\frac{(k-2) n+2 \alpha(G)+1}{2 k-2}$. Let $S=V\left(K_{3 k-2}\right), T=V\left(\frac{3 k+1}{2} K_{2}\right)$. Then $|S|=3 k-2,|T|=3 k+1$, and $d_{G-S}(T)=3 k+1$. Since $T=V\left(\frac{3 k+1}{2} K_{2}\right)$ 
is not independent, $\varepsilon(S, T)=2$. Thus, we obtain

$$
\begin{aligned}
\delta_{G}(S, T) & =k|S|+d_{G-S}(T)-k|T| \\
& =k(3 k-2)+3 k+1-k(3 k+1) \\
& =1<2=\varepsilon(S, T) .
\end{aligned}
$$

Then by Lemma 2.1, $G$ is not a fractional $k$-deleted graph. In the above sense, the condition $\delta(G)>\frac{(k-1)(n+2)+2}{2 k-1}$ in Theorem 6 is best possible.

Let $k \geq 3$ is odd. Obviously, $\frac{5 k+1}{2}$ is a positive integer. Put $G=$ $K_{3 k} \bigvee\left(2 k K_{1} \bigcup \frac{k+1}{2} K_{2}\right)$. We use $n$ to denote the order of the graph $G$. Then $n=6 k+1>4 k+1-4 \sqrt{k-1}$ and $\alpha(G)=2 k+\frac{k+1}{2}=\frac{5 k+1}{2}$. Thus, $\delta(G)=3 k=\frac{3 k(2 k-2)}{2 k-2}=\frac{6 k^{2}-6 k}{2 k-2}=\frac{(k-2)(6 k+1)+(5 k+1)+1}{2 k-2}=\frac{(k-2) n+2 \alpha(G)+1}{2 k-2}$ and $\delta(G)=3 k=\frac{3 k(2 k-1)}{2 k-1}=\frac{(k-1)(6 k+3)+3}{2 k-1}=\frac{(k-1)(n+2)+3}{2 k-1}>\frac{(k-1)(n+2)+2}{2 k-1}$. Let $S=V\left(K_{3 k}\right), T=V\left(2 k K_{1} \bigcup \frac{k+1}{2} K_{2}\right)$. Clearly, $|S|=3 k,|T|=3 k+1$, and $d_{G-S}(T)=k+1$. Since $T=V\left(2 k K_{1} \cup \frac{k+1}{2} K_{2}\right)$ is not independent, $\varepsilon(S, T)=2$. Thus, we have

$$
\begin{aligned}
\delta_{G}(S, T) & =k|S|+d_{G-S}(T)-k|T| \\
& =k \cdot 3 k+k+1-k(3 k+1) \\
& =1<2=\varepsilon(S, T) .
\end{aligned}
$$

Then by Lemma 2.1, $G$ is not a fractional $k$-deleted graph. In the above sense, the condition $\delta(G)>\frac{(k-2) n+2 \alpha(G)+1}{2 k-2}$ in Theorem 6 is best possible.

\section{References}

[1] J. A. Bondy, U. S. R. Murty, Graph Theory with Applications, London, The Macmillan Press, 1976.

[2] J. Cai, G. Liu, Stability number and fractional $f$-factors in graphs, Ars Combinatoria 80(2006), 141-146.

[3] K. Kotani, Binding numbers of fractional $k$-deleted graphs, Proceedings of the Japan Academy, Ser. A, Mathematical Sciences 86(2010), 85-88.

[4] Z. Li, G. Yan, X. Zhang, On fractional $(g, f)$-deleted graphs, Mathematica Applicata (Wuhan) 16(2003), 148-154.

[5] G. Liu, L. Zhang, Toughness and the existence of fractional $k$-factors of graphs, Discrete Mathematics 308(2008), 1741-1748. 
[6] T. Niessen, Nash-Williams conditions and the existence of $k$-factors, Ars Combinatoria 34(1992), 251-256.

[7] T. Nishimura, A degree condition for the existence of $k$-factors, Journal of Graph Theory 16(1992), 141-151.

[8] J. Yu, G. Liu, M. Ma, B. Cao, A degree condition for graphs to have fractional factors, Advances in Mathematics (China) 35(2006), 621-628.

[9] S. Zhou, A minimum degree condition of fractional $(k, m)$-deleted graphs, Comptes rendus Mathematique 347(2009), 1223-1226.

[10] S. Zhou, A result on fractional $k$-deleted graphs, Mathematica Scandinavica 106(2010), 99-106.

[11] S. Zhou, A sufficient condition for a graph to be an $(a, b, k)$-critical graph, International Journal of Computer Mathematics 87(2010), 2202-2211.

[12] S. Zhou, Independence number, connectivity and $(a, b, k)$-critical graphs, Discrete Mathematics 309(2009), 4144-4148.

[13] S. Zhou, Some new sufficient conditions for graphs to have fractional $k$ factors, International Journal of Computer Mathematics 88(2011), 484490.

Xiangyang Lv,

School of Economics and Management

Jiangsu University of Science and Technology

Mengxi Road 2, Zhenjiang, Jiangsu 212003

People's Republic of China

Email: xiangyanglv@yeah.net 
